\title{
Development of an Autofluorescence Spectral Database for the Identification and Classification of Microbial Extremophiles
}

Chandran R. Sabanayagam ${ }^{1,2,3}$, Hillari Howard ${ }^{1}$ and Richard B. Hoover ${ }^{4}$

${ }^{1}$ Center for Research and Education in Optical Sciences and Applications, ${ }^{2}$ Center for Applied Optics in Space Sciences, ${ }^{3}$ Department of Physics \& Pre-engineering, Delaware State University, Dover, Delaware 19901, ${ }^{4}$ Space Science Office, Mail Code 62, NASA/Marshall Space Flight Center, Huntsville, AL 35812

Extremophiles are microorganisms that have adapted to severe conditions that were once considered devoid of life. The extreme settings in which these organisms flourish on earth resemble many extraterrestrial environments. Identification and classification of extremophiles in situ (without the requirement for excessive handling and processing) can provide a basis for designing remotely operated instruments for extraterrestrial life exploration. An important consideration when designing such experiments is to prevent contamination of the environments. We are developing a reference spectral database of autofluorescence from microbial extremophiles using long-UV excitation (405 nm). Aromatic compounds are essential components of living systems, and biological molecules such as aromatic amino acids, nucleotides, porphyrins and vitamins can also exhibit fluorescence under long-UV excitation conditions. Autofluorescence spectra were obtained from a confocal microscope that additionally allowed observations of microbial geometry and motility. It was observed that all extremophiles studied displayed an autofluorescence peak at around $470 \mathrm{~nm}$, followed by a long decay that was species specific. The autofluorescence database can potentially be used as a reference to identify and classify past or present microbial life in our solar system. 